www. revis tad y o. com

\title{
Oportunidades dentro de los procesos de mundialización textil internacio- nal y relación con la RSE a través de un analisis DELPHI: ética o estética ${ }^{1}$
}

Opportunities within the processes of globalization in the textile sector and their relation to CSR through a DELPHI analysis: ethical or aesthetic

Arturo Luque González¹, Juan Hernández Zubizarreta² y Carmen de Pablos Heredro

${ }^{1}$ Universidad Rey Juan Carlos de Madrid. E-mail: arturo@elcandelero.es

2 Universidad del País Vasco. E-mail: juan.hernandez@uhu.eus

${ }^{1}$ Universidad Rey Juan Carlos de Madrid. E-mail: carmen.depablos@urjc.es

Fecha de recepción: 01-07-2016

Fecha de aceptación: 19-07-2016

Resumen: El sector textil internacional ha evolucionado en todas las direcciones aprovechándose de los recursos existentes en cada momento para crecer. Desde aproximadamente los años 1990 se empiezan a desarrollar sin precedentes los sistemas de transporte y telecomunicación hasta hoy en día donde la comunicación telemática de carácter autónomo es el proceder diario de la producción y sus cadenas de valor globales. Se coordinan miles de proveedores y millones de clientes de manera simultánea generando un torrente de información que permite tomar decisiones más ajustadas. Desde una perspectiva crítica, basada en el análisis de la literatura precedente, en este trabajo se ha contado con la participación de 55 expertos donde a través de un análisis Delphi, se han analizado oportunidades del sector.

Palabras clave: Globalización, responsabilidad social corporativa, empresas transnacionales, oportunidades, industria textil.

\footnotetext{
1 Este proyecto de investigación nunca podría haberse realizado sin la aportación de todos los expertos que han colaborado de manera altruista y desinteresada aunque no por ello con menor dedicación a tenor de las casi 450 hojas de respuestas -cuantitativas y cualitativasrecibidas de los participantes objeto de discusión académica. Destacando a Fernando Oliván (director del observatorio Euromediterráneo, ex-miembro de la CPI y profesor de la URJC), Isidor Boix (secretario de Acción Sindical Internacional de FITEQA-CC.OO), Octavio Granado (experto en financiación pública y ex secretario de Estado de Seg. Social), Michael Addo (investigador en la universidad de Exeter, Devon, Reino Unido y miembro del Grupo de Trabajo sobre Empresas y Derechos Humanos de las Naciones), Gabriel Flores Sánchez (investigador del Instituto Complutense de Estudios Internacionales), Aleida Hernández (investigadora de la Universidad Nacional Autónoma de México), Juan Andrés Cano (CEO de Value4Chain), María del Mar Maira Vidal (Investigadora en la Universidad de Valladolid), Rodrigo Martín (Investigador en la URJC, ex magistrado del TSJM y miembro del Consejo Académico de Gómez-Acebo \& Pombo), Alejandro Goldberg (investigador en CONICET), Amaya Apesteguía (OCU), Ibon Maza (BabyAuto), Enrique Dussel (investigador de la Universidad Nacional Autónoma de México y coordinador de estudios en CEPAL y OIT), Jesús García Luengos (Coordinador del think tank RESET Research on Security and Transnational Governance), Jordi Bonet (Catedrático de la UB en derecho internacional público), José Manuel Pureza (investigador en la Universidad de Coímbra Y CES, arbitro CPA y de Naciones Unidas sobre el mar), Roberto Toscano (investigador de relaciones internacionales y RSE, además de ex embajador de Italia en India e Irán), Juan Manzanedo (Ceo de LOGISFASHION), Juan Pérez Ventura (Ceo de El Orden Mundial en el S. XXI), Paula Alves (empleada de Inditex desde hace 24 años y Secretaria del Sector Textil-Piel de FITAG-UGT), Santi Mallorquí (Ceo de ORGANIC COTTON COLOURS), Martim Gemzell (WARONTWANT), Gema Gómez (Ceo SLOW FASHION SPAIN), Carmen Gómez-Cotta (ETHIC), Enrique Palazuelos Manso (catedrático de economía aplicada en la Universidad Complutense de Madrid), Michael Tamvakis (investigador en Cass Business School), Eva García (ECOOLOGY), Alejandro Dulitzky (investigador en Universidad Nacional de San Martín), Juan Torres (Catedrático de Economía Aplicada en la Universidad de Sevilla y miembro del comité científico de ATTAC), Amparo Merino Segovia (investigadora en Universidad de Castilla la Mancha), Gonzalo Berrón (FES Fundación Friedrich Ebert), Alejandro Teitelbaum (escritor, abogado y representante de la Federación Internacional de Derechos Humanos y de la Asociación Americana de Juristas ante NACIONES UNIDAS), Carmen Silla (JEANOLOGIA), Antonio Baylos (catedrático de Derecho del Trabajo y de la Seguridad Social en la Universidad de Castilla la Mancha), Álvaro Orsatti (CSA), Monika Kemperle (IndustriALL Global Union), Mariano Aguirre (director del Norwegian Peacebuilding Resource Centre NOREF en Oslo), Pablo José Martínez Osés (investigador y coordinador de la Campaña estatal Pobreza Cero y miembro de 2015ymas), Iñaki Barcena (catedrático de Ciencia Política en la Universidad del País Vasco), Virginia Rondeel (Moda Sostenible BCN), Marta Castells (CITYC), Enrique Guerrero Salom (profesor y eurodiputado), Javier Chércoles (investigador en la Universidad de Dhaka y director de riesgos en Associated British Foods plc), Esteban Kaipl (investigador en Universidad de Litoral), Iratxe Arteagoitia \& Eva Kreisler (SETEM \& CLEAN CLOTHES CAMPAIGN), José Miguel Mulet (Investigador del Instituto de Biología Molecular y Celular de Plantas IBMCP de Valencia), Amanda Cattermole (CATTERMOLECONSULTING), Liliane Spendeler (AMIGOS DE LA TIERRA), Cheryl S. Watson (investigadora en University of Texas Medical Branch), Isabel Soriano (AITEX), Dolores Romano (investigadora independiente/Ecologistas en acción), Antonio Solé Cabanes (ASOLEGIN), Oscar Miralles (investigador independiente), Xavier Giménez Font (investigador de Química Ambiental en la Universidad de Barcelona), Raimon Guitart (investigador en toxicología en Universidad Autonoma de Barcelona), Ferran Ballester (coordinador del área de Ambient i Salut del Centro Superior de Investigación en Salud Pública CSISP), Stephane Horel (independiente) y Carlos de Prada (Fodesam)
} 


\begin{abstract}
The international textile sector has evolved in all directions, taking advantage of existing resources to grow. Since the 1990s it started to be an unprecedented transport and telecommunications systems development that has become a source of opportunities where distance on line autonomous communication is the cornerstone of everyday production. Thousands of suppliers and millions of customers are coordinated simultaneously generating great quantity of information that helps promoting a more adjusted decision making. Form a critical perspective based in the literature review, in this research 55 experts have participated in a Delphi analysis oriented to better know the opportunities of the industry.
\end{abstract}

Keywords: Globalization, corporate social responsibility, transnational companies, opportunities, textile industry.

\section{Introducción}

Uno de los cambios más determinantes sufridos por la humanidad a nivel económico, social y laboral, es la globalización que ha condicionado todo tipo de políticas internas y externas.

En época de la guerra fría se escenificó mediante el telón de acero, actualmente se ejemplifica mediante el desarrollo de un sistema en red interconectado en el cual a través de todo tipo de relaciones, se generan oportunidades, aunque no para todos igual.

"La globalización significa los procesos en virtud de los cuales los Estados nacionales soberanos se entremezclan e imbrican mediante actores transnacionales y sus respectivas probabilidades de poder, orientaciones, identidades y entramados varios. El concepto de globalización se puede describir como un proceso, que crea vínculos y espacios sociales transnacionales, revaloriza culturas locales y trae a un primer plano terceras culturas..." (Beck, 1998, p. 29).

La integración de sistemas de comunicación permite la conectividad en tiempo real dando soporte a la automatización de la actividad productiva ${ }^{2}$. La creación de sofisticados sistemas de información y gestión empresarial posibilitan el desarrollo de elementos autónomos interconectados a tiempo completo que junto al empuje de nuevos productos textiles (prendas técnicas, mejoras en prendas de seguridad debido a la incorporación de legislaciones más estrictas como prendas ignifugas, etc) inciden positivamente en la producción textil.
Las prendas textiles contienen gran cantidad de productos químicos que junto a nuevos métodos de producción y distribución, generan clara fuente de innovación, competitividad y crecimiento a nivel global (también se generan otra serie de asimetrías como residuos y producción-demanda). Estos productos son utilizados por los consumidores y promocionados por las empresas transnacionales (ET) ávidas de consumo y de necesidades -en muchos casos creadas artificialmente- fomentando el leit motiv empresarial de las empresas textiles. La expansión de las ET apoyadas en la libre circulación de productos y capitales ha conseguido crear una superestructura a nivel global a la carta donde en relación a los requerimientos deseados por el cliente, se puede conseguir prácticamente a medida cualquier producto, servicio y precio. Los costes tanto del transporte como de la fuerza del trabajo se han desplomado. Se fijan tiradas ${ }^{3}$ de prendas que se desean realizar y el precio máximo disponible a pagar por ellas sin importar si se realizan en Marruecos, Brasil o Vietnam. Circunstancia que es aprovechada por las ET junto a más medidas como los tratados bilaterales, de libre comercio y zonas francas (objeto de fiscalidad a medida) a su servicio. Para (Toscano, 2015) "Una vez (en los famosos años 60) se atacaba al capitalismo como si fuera vinculado a la austeridad, al sacrificio, a la disciplina, a la represión. Colosal equivocación. Hoy la fuerza del capitalismo reside en su capacidad de proporcionar (por supuesto, no a todos) consumo, gozo, entretenimiento. Mi generación atacaba el Capitalismo-Caresma y ahora estamos en el Capitalismo-Carnaval".

\footnotetext{
2 "Desde la gestión de la relación con los clientes (CRM) hasta planificación de recursos empresariales (ERP), desde la gestión de la cadena de suministros hasta el business intelligence y el reporting, Microsoft Dynamics tiene la solución de empresarial adecuada para usted.Y cuando se combina Microsoft Dynamics con Office, Skype y Yammer, puede conectarse al instante con sus equipos. Con Microsoft Dynamics, puede añadir colaboración y productividad a cualquier dispositivo, en cualquier lugar" Información extraida de la página oficial de Microsoft Dynamics el 02/12/2015.

3 "Así China es especialmente competitiva para grandes series de productos estándar, como por ejemplo pantalones, pero su competitividad es menos evidente para series cortas, productos más especializados o para la pronto moda. "Según el informe Previsiones sobre la evolución del sector textil/confección en el horizonte de 2015, p. 14, elaborado por el Observatorio Industril del Sector Textil/Confección.
} 
En este contexto las ET textiles han sabido adaptarse externalizando todos los costes con afán de ser reducidos utilizando todas las posibilidades existentes a nivel legislativo, logístico y empresarial (OIT, 2015), lo que ha permitido que hoy en día pueda tener un coste superior la cremallera de un pantalón en una tienda de occidente que el propio pantalón producido en cualquier rincón de Asia. Se han desarrollado nuevos modelos productivos asentados en subcontrataciones, deslocalizaciones, externalizaciones o relocalizaciones de toda la cadena de valor. Para (Harbulot, 2012, p. 157) "La calidad de los productos, la insuficiente formación de los trabajadores locales, las fluctuaciones del precio de transporte vinculadas a las fluctuaciones del petróleo y los costes funcionales que conlleva la distancia geográfica obligan a las empresas a relocalizar sus actividades" circunstancia esta que permite igualmente identificar sus ventajas competitivas ante otros competidores pese a estar dividido ese conocimiento y producción entre miles de proveedores y sistemas de información. Cabe señalar que los acuerdos de outsourcing no se declaran en los balances contables ya que no llevan aparejada la necesidad de hacer una provisión. Los costes de reestructuración se producen en el año contable y se tratan como un gasto periódico en lugar de como una obligación a largo plazo ${ }^{4}$.

En la siguiente tabla $\mathrm{n}^{01}$ Estadísticas de externalización, podemos observar un amplio resumen estadístico sobre la incidencia en cifras concretas de la externalización en relación a diferentes usos y mercados.

Tabla n¹. Estadísticas de subcontratación

\begin{tabular}{|c|c|c|}
\hline \multicolumn{3}{|c|}{ ESTADÍSTICAS DE SUBCONTRATACIÓN } \\
\hline $\begin{array}{l}\text { Número total de puestos de trabajo que Estados Unidos ha subcontratado } \\
\text { en } 2015\end{array}$ & \multicolumn{2}{|c|}{$2,382,000$} \\
\hline Número de puestos de trabajo subcontratado a China desde 2001 & \multicolumn{2}{|c|}{$3,200,000$} \\
\hline $\begin{array}{l}\text { Porcentaje de directores financieros encuestados que afirmaban que sus } \\
\text { firmas subcontrataban en el extranjero }\end{array}$ & \multicolumn{2}{|c|}{$38 \%$} \\
\hline $\begin{array}{l}\text { Porcentaje de directores financieros encuestados que afirmaban que sus } \\
\text { firmas favorecían la subcontrataban en China }\end{array}$ & \multicolumn{2}{|c|}{$18 \%$} \\
\hline Porcentaje de directores financieros encuestados que afirmaban que sus & \multicolumn{2}{|c|}{$26 \%$} \\
\hline $\begin{array}{l}\text { RANKING DE PROFESIONES CON RIESGO DE SUBCONTRATACIÓN } \\
\text { EN } 2015\end{array}$ & EMPLEOS PERDIDOS & SALARIOS PERDIDOS \\
\hline Ingenieros informáticos/ ingenieros de software & 211,700 & $\$ 14,400.000 .000$ \\
\hline Contables / auditores & $\begin{array}{c}160,000 \\
8,500,000,000\end{array}$ & $\$ 8,500,000,000$ \\
\hline Abogados & 40,400 & $\$ 4,300,000,000$ \\
\hline Agentes de venta de seguros & 11,000 & $\$ 591.000 .000$ \\
\hline Corredores de Bienes inmuebles / agentes & 6,700 & $\$ 332$ \\
\hline Químicos / físicos & 3,700 & $\$ 230$ \\
\hline
\end{tabular}

\footnotetext{
${ }^{4}$ Según Financial Report Standard número doce en el caso de Uk. https://www.frc.org.uk/Our-Work/Publications/ASB/FRS-12-Provisions,-Contingent-Liabilities-and-Cont/FRS-12-Provisions,-Contingent-Liabilities-and-Cont.aspx
} 


\begin{tabular}{|c|c|c|c|c|}
\hline \multicolumn{2}{|c|}{ PORCENTAJE DE COMPAÑÍAS QUE EXTERNALIZAN POR SECTOR } & \multicolumn{3}{|c|}{ DE COMPAÑÍAS } \\
\hline \multicolumn{2}{|l|}{ Manufacturas } & \multicolumn{3}{|c|}{$53 \%$} \\
\hline \multicolumn{2}{|l|}{ Servicios técnicos } & \multicolumn{3}{|c|}{$43 \%$} \\
\hline \multicolumn{2}{|l|}{ Investigación y desarrollo I+D } & \multicolumn{3}{|c|}{$38 \%$} \\
\hline \multicolumn{2}{|l|}{ Distribución } & \multicolumn{3}{|c|}{$26 \%$} \\
\hline \multicolumn{2}{|l|}{ Centros de asistencia telefónica (call centers) } & \multicolumn{3}{|c|}{$12 \%$} \\
\hline \multicolumn{2}{|c|}{$\begin{array}{l}\text { RAZONES POR LAS CUALES LAS EMPRESAS SUBCONTRATAN } \\
\text { (MÚLTIPLES RESPUESTAS PERMITIDAS) }\end{array}$} & \multicolumn{3}{|c|}{ PORCENTAJE } \\
\hline \multicolumn{2}{|l|}{ Reduce el control de costes } & \multicolumn{3}{|c|}{$44 \%$} \\
\hline \multicolumn{2}{|c|}{ Obtener acceso a recursos técnicos no disponibles internamente } & \multicolumn{3}{|c|}{$34 \%$} \\
\hline \multicolumn{2}{|l|}{ Liberar recursos internos } & \multicolumn{3}{|c|}{$31 \%$} \\
\hline \multicolumn{2}{|l|}{ Mejorar el negocio o enfocarse al cliente } & \multicolumn{3}{|c|}{$28 \%$} \\
\hline \multicolumn{2}{|c|}{ Acelerar la reorganización/transformación de la empresa } & \multicolumn{3}{|c|}{$22 \%$} \\
\hline \multicolumn{2}{|l|}{ Aceleración de proyectos } & \multicolumn{3}{|c|}{$15 \%$} \\
\hline \multicolumn{2}{|c|}{ Tener acceso a la experiencia en la gestión disponible internamente } & \multicolumn{3}{|c|}{$15 \%$} \\
\hline \multicolumn{2}{|l|}{ Reducir el tiempo de comercialización } & \multicolumn{3}{|c|}{$9 \%$} \\
\hline \multicolumn{2}{|c|}{$\begin{array}{l}\text { QUE PIENSAN LOS ECONOMISTAS SOBRE LA INCIDENCIA DE LA } \\
\text { EXTERNALIZACIÓN EN EL EMPLEO }\end{array}$} & \multicolumn{3}{|c|}{ PORCENTAJE } \\
\hline \multicolumn{2}{|l|}{ Daña a la economía } & \multicolumn{3}{|c|}{$89 \%$} \\
\hline Ayuda a la economía & & & $17 \%$ & \\
\hline No tiene efectos & & & $10 \%$ & \\
\hline No estoy seguro & & & $4 \%$ & \\
\hline $\begin{array}{l}\text { LISTADO DE PAÍSES MEJOR VALORA- } \\
\text { DOS PARA EXTERNALIZAR }\end{array}$ & CALIFICACIÓN GENERAL & $\begin{array}{l}\text { ÍNDICE DE } \\
\text { COSTES }\end{array}$ & $\begin{array}{l}\text { RECURSOS } \\
\text { HABILIDADES }\end{array}$ & FUERZA DEL TRABAJO \\
\hline India & 7.1 & 8.3 & 6 & $1,430,000,000$ \\
\hline Indonesia & 6.9 & 8.6 & 4.3 & $1,033,000,000$ \\
\hline China & 6.4 & 7 & 5.6 & $780,000,000$ \\
\hline Bulgaria & 6.4 & 8.8 & 2.9 & $3,000,000$ \\
\hline Filipinas & 6.3 & 9 & 2.8 & $39,000,000$ \\
\hline
\end{tabular}




\begin{tabular}{|l|c|c|c|c|}
\hline Jordania & 6.2 & 7.6 & 2.7 & $2,000,000$ \\
\hline Singapur & 6.5 & 6.4 & 5.7 & $3,000,000$ \\
\hline Tailandia & 6 & 8.2 & 2.3 & $39,000,000$ \\
\hline Lituania & 5.9 & 7 & 3.9 & $2,000,000$ \\
\hline Egipto & 5.8 & 9 & 0.9 & $26,000,000$ \\
\hline Malasia & 5.8 & 7.9 & 2.2 & $12,000,000$ \\
\hline Estonia & 5.8 & 7.5 & 5.2 & $1,000,000$ \\
\hline Chile & 5.7 & 7.2 & 3 & $8,000,000$ \\
\hline Hungrí & 5.6 & 6.9 & 3.4 & $4,000,000$ \\
\hline
\end{tabular}

Fuente: Sourcing Line Computer Economics, Associated Press a través de Statistic Brain con fecha de la investigación 6/10/2015

Hoy en día un individuo puede ser dueño de una empresa con miles de trabajadores y millones de beneficios sin poseer instalaciones, maquinaria propia, trabajadores por cuenta propia, etc., además de poder trabajar en Europa, tener radicada la sociedad en cualquier país del mundo con mejores condiciones fiscales y operar desde centenares de lugares al utilizar servicios modulares ad hoc bajo demanda y a modo de banderas de conveniencia societarias como hacen muchos mercantes (Meyers, 2012).

Es indudable que la evolución industrial de corporaciones multinacionales y empresas transnacionales (ET) textiles también han traído consigo un aumento de riqueza para la sociedad de muy diversas formas -y desigual incidencia- en definitiva, un crisol de oportunidades para millones de ciudadanos y países sin precedentes (letto-Gillies, 2012) y (Lovins, Hunter, \& Hawken, 2013). Cabe señalar que en la actualidad debido al ciclo económico adverso que atraviesan infinidad de estados y pueblos, además de situaciones de difícil explicación como son el trabajo infantil ${ }^{5}$, condiciones inhumanas para trabajadores desveladas por ONG's, corrupción, etc., se ponga en evidencia la manera de producir de determinadas ET amparadas por determinados gobiernos y entidades supranacionales.
Entrando más en detalle podemos destacar los siguientes beneficios a nivel organizativo de una ET textil, que desarrolla actividad en otro estado como son:

1) Generación de ofertas de trabajo en los lugares donde operan (y territorios colindantes), pudiendo contribuir a un mayor dinamismo y una fuente de riqueza en esos lugares (Tokmann, 2012)

2) Desarrollo de fuentes de financiación propias con capacidad autónoma de ejecución, desarrollando a su vez un sistema integral formado por las formas y tipos más variados de capital (Antolín \& Cendrero, 2011), 3) Posibilidad de gestionar sinergias nacionales e internacionales con motivo de poseer una gran capacidad económica así como experiencia tanto con gobiernos, sociedad o proveedores (Pulido \& Ramiro, 2011), 4) Con motivo de tender continuamente hacia una mejor producción, se desarrolla de manera directa y colateral un I+D+I aplicable a muchos otros sectores generando una mayor riqueza de manera indirecta (Moro, 2011), 5) Potenciación de la marca, siendo en muchos casos conocida internacionalmente y respaldada por lo que está en ventaja sobre la producción autóctona que puede fabricar incluso un producto igual o mejor (Hernández, Ramírez , \& Álvarez, 2013), 6)

\footnotetext{
${ }^{5}$ En muchos casos es admitido por IT y por Unicef atendiendo a su desarrollo físico, emocional y siempre que sea compatible con su escolarización, la OIT recoge y regula su aproximación en las convenciones número 138 y 182.
} 
Capacidad de maniobra de una ET textil. Siendo esta tan grande, hace que sus precios sean muy competitivos y en muchos de los casos inigualables — de ahí que muchas empresas recurran a competir en calidad o mediante dumping vinculando esa pérdida con la de la compra de otro producto que si genere beneficio(Ríos Núnez \& Coq Huelva, 2010), 7) La irradiación económica por el establecimiento de una actividad industrial o acuerdo en un territorio concreto puede ser grande, tanto para los propios empleados como para empresas de las que se nutre (Bouzas, 1994), 8) Con motivo de más desarrollo y capacidad, aumenta el catálogo de productos y sinergias (Larrea, 2013), 9) En muchos casos ayuda a las relaciones entre gobiernos tanto económicas como diplomáticas debido a un interés común (Vargas Hernández, 2012), 10) Los accionistas e inversores pueden tener una mayor capacidad de maniobra al poder operar en diferentes países, mercados o productos - también puede tener consecuencias negativas-(Grau-Ruiz, 2013), 11) Orientación a la hora de satisfacer la demanda del mercado interno de los países en donde actúan sus filiales -inclinándose por los mercados más grandes- (Dunning, 2012),

12) Optimización en la asignación de recursos como señala (Rugman, 1982), 13) Gestión y circulación interna de conocimiento y 14) Mayor capacidad de renegociación de deudas o problemas financieros (Kalmanovitz, 2010).

Además de lo anteriormente expuesto, existen otras fuentes de ventajas hoy ya latentes como ejemplificadas por el cambio de paradigma productivo asentado en la cobertura de necesidades reales, sostenibilidad, respeto (interno y externo) y reciclaje, los cuales poco a poco se irán imponiendo pese a la existencia de intereses opuestos por parte de las grandes corporaciones -y gobiernos - . A modo de ejemplo es "entendible" que el lobby eléctrico no favorezca cualquier tipo de acción sostenible en favor de energías alternativas o autoconsumo solar si pueden verse afectados sus beneficios, circunstancia que puede ser entendible pero no admisible por parte de la sociedad en defensa propia de sus intereses $-\mathrm{y}$ de manera delegada del medio ambiente-.
El modelo de sociedad actual se basa en el crecimiento. Continuamente se están desarrollando nuevos bienes y servicios capaces de satisfacer y generar nuevas necesidades, como señala el director de la unidad de Psicología y Consumidor de la Universidad de Valencia (Quintanilla, 2015) "Al final, nos comportamos así porque conviene que nos comportemos así, porque para que el modelo funcione el consumo no puede parar". Podíamos decir que se crean necesidades sistémicas, según (OrganicCottonColours, 2015) "El modelo capitalista tiene que mejorar y la solución creo que sería una nueva economía del bien común". Cada vez adquieren mayor resonancia las voces que señalan la necesidad de crear una producción más sostenible y justa. Pero cabe preguntarse, ¿se puede ser competitivo y a la vez sostenible?, ese es un dilema que debe responder la clase empresarial internacional ya que la sociedad ha contestado a través de sus actos, demandas - y anémicos sufragios-, o ¿por qué es necesario cambiar parte del modelo productivo [por parte de la industria] si ya existe tecnología, se generan beneficios y se cumple la normativa vigente? Es ahí donde entran en juego los nuevos contrapoderes sociales como son las ONG's, librepensadores, estudios independientes - sin necesidad de cobertura empresarial-, con la finalidad de erradicar procesos consustanciales de corrupción unidos a toda actividad comercial (Tanzi \& Davoodi, 1997) apoyados en muchos casos en legislaciones débiles, con la finalidad de evitar situaciones de necesidad (Human Rights Watch, 2015).

Las circunstancias anteriormente señaladas deben suponer un punto de inflexión a la hora de producir de una manera ética, además de generar un conjunto de procedimientos y posibilidades empresariales a través de la inclusión de sociedades desfavorecidas. Estas situaciones aumentarán la reputación ${ }^{7}$ y los beneficios pudiendo dejar las campañas de impacto — para otros menesteres - centrándose en lo que realmente importa. Hoy en día más del $84 \%$ de la valoración ${ }^{8}$ de una empresa en el mercado de valores se basa en intangibles. Es decir, que factores como reputación, capital humano, relación con el entorno, pesan más que los activos mismos. El mercado comprende que estas son las bases para la existencia a largo plazo. Como señala (Miralles, 2015) "En una sociedad en la que el poder político no toma las decisiones adecuadas, el consu-

\footnotetext{
${ }^{6}$ El Gobierno legisla al dictado del lobby energético señalando que "El mercado eléctrico está dominado por oligopolios con una gran presencia de la banca, sin apenas control público, y con una gran influencia sobre las leyes que regulan el sector" información elaborada por M. Cappa a través de LaMarea.com del 4/11/2013.

-En Marzo de 2015 el Lobby eléctrico Eurelectric ha elaborado un informe titulado A reference model for european capacity markets en el cual se exige total libertad para operar y por tanto legislar el 100\% de las cuestiones energéticas dentro de la UE.

${ }^{7}$ La reputación es definida por Charles Fombrum miembro del "Reputation Institute", como aquella medida en la que una empresa es admirada y respetada

${ }^{8}$ Oceantomo, empresa de Capital Intelectual Merchant Banc TM proporciona servicios financieros relacionados con los activos intangibles de la industria, testimonios de expertos, valoración, estrategia, investigación, calificaciones, inversiones, gestión de riesgo y transacciones.
} 
midor sigue consumiendo productos de empresas con dudosa reputación, sin ningún tipo de concienciación, todo esto unido a empresas cuyos datos económicos son más importantes que cualquier otro objetivo. ¿Qué parte debe asumir cada uno?".

Se han generado nuevos cánones de solidaridad articulándose muchos de ellos a través del poder emergente del consumidor mediante sus quejas y rechazos en las redes sociales y el uso o no de su tarjeta de crédito como veredicto. Con ello se han articulado otras fuentes de control externo empresarial -ante la renuncia de muchos gobiernos a correctas regulaciones - mediante una mejora continua como es el consumo colaborativo (aumentando la competencia aunque de manera limitada). Según el análisis de (Reinecke \& Donaghey, 2015, p. 35) "la unión de diferentes campañas junto a la acción sindical, coordinaron una respuesta unificada ante el desastre de Rana Plaza en Bangladesh del año 2013 [donde murieron más de 1100 personas], sostenemos que la intersección de la producción y la fuerza del consumo, que ha recibido poca atención hasta la fecha, es un mecanismo potente que puede fomentar los derechos laborales en las cadenas de suministro globales." sin olvidar como indican (Folkes \& Kammis, 1999) que el consumo responsable no sustituye la calidad del producto que se adquiere y tampoco una calidad superior del mismo compensa el tener comportamientos no éticos. Muchos consumidores demandan nuevas características en sus prendas textiles siendo estas prácticamente a medida en cuanto a producción, diseño, componentes y entrega ${ }^{9}$. Todas estas características deben ser cumplidas con la mayor diligencia posible para no persuadir al consumidor de su compra según el informe sobre las previsiones sobre la evolución del sector textil/confección en el horizonte 2015 (Observatorio Industrial del Sector Textil/Confección, 2015, p. 14).

El modelo de sociedad y economía actual lleva consigo una ecuación desigual para todos los ciudadanos del planeta y en concreto para los diferentes países en vías de desarrollo. Las empresas se sienten atraídas por la mano de obra barata de los países subdesarrollados, "La principal atracción de la subcontratación en el extranjero es la reducción de costes. Las retribuciones al personal que se satisfacen en la India, aunque resulten relativamente razonables para el nivel habitual en dicho país, representan una pequeña fracción de los salarios abonados en occidente" ${ }^{\prime 0}$. Cabe destacar el último informe mundial sobre salarios 2012 / 2013 de la OIT (salarios y crecimiento) a través de una correlación de productividad con los salarios "La existencia de un gran excedente en la cuenta corriente de algunos países, indica que existe espacio para estimular la demanda doméstica, principalmente mediante una mejor vinculación de los aumentos de la productividad y de los salarios" (OIT, 2013, p. 84) circunstancia relacionada de manera directa con los derechos humanos. Las ET deben mantener una posición proactiva que genere igualdad y seguridad, en relación a los derechos humanos $^{11}$, para (Fernando \& Rivera, 2013, pp. 331-335) "[...] las empresas se verán favorecidas en un entorno en el que el cumplimiento de los derechos humanos sea la norma, y no la excepción", circunstancia que más allá de ser obligatoria abre una nueva vía de negocio y credibilidad.

\section{Metodología}

Para la elaboración de este análisis se han utilizado dos técnicas de investigación que conjugan la perspectiva cualitativa y cuantitativa como son la entrevista y la técnica Delphi (Ruiz Olabuénaga \& Ispuzua, 1989) y (Del Rincón, Arnal, Latorre, \& Sans, 1995) hacia dos paneles de expertos.

La técnica de la entrevista, que en nuestro caso se contesta de forma escrita, nos permitió conocer en detalle el punto de vista de dichos participantes (Strauss \& Corbin, 1990) sintetizándose posteriormente en un DAFO (debilidades, amenazas, fortalezas y oportunidades) en este caso a través de sus oportunidades.

\footnotetext{
9 "Los envíos vía drone ya estan aquí —y funcionan-" Los drones no necesitan conductores, no se quedan atrapados en el tráfico. "Es mucho más económico -costes de energía- y eficiente en tiempo, enviar [una muestra de sangre en el ámbito sanitario] a través de aviones no tripulados, en lugar de enviarlas en vehículos de dos toneladas de peso por carretera con una persona dentro [...]" según A. Raptopoulos, fundador y CEO de Matternet. Noticia elaborada por S. French a través de Marketwatch el 15/12/2015.

${ }^{10}$ Revista Trabajo No. 47 de la OIT, junio 2003 pags. 14 y 15.

${ }^{11}$ Existe gran cantidad de normativa no vinculante jurídicamente hablando pero recomendable seguimiento y aplicación como es el caso de "Las Directrices de la Organización para la Cooperación y el Desarrollo Económico (OCDE) para las empresas multinacional de 1976", "La Declaración tripartita de principios sobre las empresas multinacionales y la política social de la Organización Internacional del Trabajo (OIT), de 1977", "El Pacto Mundial de las Naciones Unidas (UN Global Compact) del 2000", "Los Estándares de desempeño de sostenibilidad social y ambiental de la Corporación Financiera Internacional (IFC), del 2006”, "La Guía de Responsabilidad Social, ISO 26000, de la Organización Internacional de Estandarización (ISO), del 2010", etc.
} 
El conocimiento debe de ser investigado y puesto a disposición de la sociedad y empresas a través de todo tipo de procedimientos (Arias, 2003) que permitan argumentar con calidad suficiente sus incoherencias así como en este caso sus oportunidades teniendo en cuenta la transversalidad de áreas que interconecta, en este caso el sector textil a nivel global, inclusive en áreas de incertidumbre o de falta de evidencia empírica como señala (Pill, 1971).

\section{El método DELPHI}

El método Delphi es un procedimiento prospectivo que tiene la finalidad de recabar información a través de la participación de un grupo de expertos, teniendo como base la discusión de un problema definido como es nuestro caso "en qué condiciones se produce la gestión y producción textil en el marco de países con vías de desarrollo así como la afectación propia por el propio desarrollo de la actividad allí radicada y la relación de oportunidades que se generan".

Para (Linstone \& Turoff, 1975) es un método de estructuración de un proceso de comunicación grupal permitiendo a un grupo de individuos, como un todo, tratar un problema complejo. Uno de los principios del cual partimos considera que existe mayor fiabilidad en las soluciones colegiadas (Mengual, 2011, p. 146), siendo además idóneo para el estudio de temáticas en las cuales la obtención de información tanto del pasado como del futuro no se encuentre de forma clara (Vélez Pareja, 2002).

Para (Luna Huertas, Infante Moro, \& Martínez , 2006, p. 94) "La técnica Delphi, a diferencia de otras técnicas cualitativas tales como los grupos focales o los grupos nominales, permite obtener información y opiniones de sujetos físicamente alejados, y posibilita la generación de ideas con respuestas abiertas, de forma bien estructurada y con un componente cualitativo añadido".

\section{PROCESO SEGUIDO}

Después de haber definido el problema como hemos señalado anteriormente, se acude a los expertos participantes elegidos previamente en relación a su especial relevancia debido al grado de experticia, publicaciones, áreas de investigación, responsabilidades así como capacidad de respuesta y dependencia en relación a sus organizaciones intentando evitar departamentos de comunicación ya que no era objeto de estudio el análisis de información/respuestas prefabricadas.

Las preguntas, se han estructurado y precisado teniendo en cuenta los diferentes ejes temáticos a analizar siendo estas directas, indirectas ${ }^{12}$, de investigación, específicas, estructuradas, clave, etc., "[...] traduciendo las cuestiones de investigación (objetivos, hipótesis...) en preguntas [...]" (Valles, 2014, pp. 68-71), inclusive recibiendo información muy valiosa no preguntada, relacionando las mismas a los factores que son necesarios para articular las oportunidades del sistema de producción textil internacional.

Una vez realizados los procesos anteriormente descritos, se enviaron los cuestionarios a los participantes, como señala (Martínez Piñeiro, 2003, p. 452) "El investigador elabora a partir de las respuestas recibidas el segundo cuestionario, muchas veces ya de respuestas cerradas que posibilitan un tratamiento estadístico de las respuestas del grupo", no existiendo un número de consultas fijo o preconcebido (Fernández-Ballesteros, 1995)

\section{MODELO DE CUESTIONARIO}

El cuestionario está dividido en dos partes conjugándose la recopilación de datos cuantitativos -expuesto en otro documento- y cualitativos ${ }^{13}$ objeto de esta investigación. Los primeros dirigidos a la búsqueda del consenso entre los participantes en cada panel, los segundos y objeto de este trabajo, obtenidos a través de las preguntas abiertas con el objetivo de profundizar en el punto vista de cada panelista sobre el sector textil desde diferentes prismas y conocimiento ateniéndonos a las oportunidades del sector textil.

La necesidad de conjugar lo cuantitativo y cualitativo se debe a complejidad y el dinamismo la temática a analizar, el sector textil global así como por la relevancia de la muestra obtenida por parte de los 55 participantes seleccionados. En el proyecto participan expertos en $\mathrm{RSE}$, sector químico-riesgos, gestores de producción, logística, economistas, legisladores, expertos en lobbies y empresas transnacionales, juristas, ONG's, laboratorios, divulgadores científicos, expertos en sector

\footnotetext{
12 Wengraf, 2001:63, enseña sus cartas cuando afirma que "las preguntas indirectas son a menudo mejores que las preguntas directas, y las no-preguntas (non-questions) pueden a menudo ser mejores que las preguntas indirectas". A través de la publicación Entrevistas cualitativas elaborado por M. S. Valles, de CIS-Centro de Investigaciones Sociológicas V.32, del año 2014 p.68.

${ }^{13}$ Las preguntas abiertas en forma de cuestionario o entrevista en profundidad son una metodología cualitativa válida para la recogida de información para la investigación.
} 
energético, transporte, gestores sociales, políticos, conocedores de fenómenos migratorios, negociación colectiva, etc., por lo que se decidió acotar la temática de los mismos en dos paneles de expertos $1^{\circ}$ expertos en RSC, gestión, ética, procesos de mundialización, etc., y $2^{\circ}$ expertos en epidemiología, laboratorios, científicos químicos y expertos en salud, incluyendo a su vez preguntas multidisciplinares $-\mathrm{y}$ comunes a ambos cuestionarios - en las cuales el panel de participantes pudiera responder debido a su conocimiento.

El primero de los cuestionarios consta de 15 preguntas abiertas orientadas ver anexo I a la RSE, globalización, corrupción, ética empresarial, protección de los trabajadores, riesgos, empuje del consumidor, ONG's, etc., a través de un modelo de entrevista escrita basándonos en la literatura existente así como en entrevistas personales realizadas con otros expertos y profesionales no participantes.

El segundo de ellos ha sido elaborado con 11 preguntas abiertas orientadas a expertos en riesgos químicos, tóxicos, agencias de evaluación, tintes textiles, etc., con la salvedad de que 4 de las abiertas eran comunes entre ambos ya que además de tener capacidad suficiente para su respuesta, generaba profundidad a la hora de analizar las respuestas.

Después de una primera ronda de cuestionarios enviados y recibidos se analizaron de manera cualitativa las respuestas dadas a las 15 preguntas abiertas de expertos en RSE, legislación, globalización, representación colectiva de trabajadores así como las 11 preguntas abiertas realizadas a expertos químicos y riesgos, para ser añadidas sus consideraciones - previo análisis - en conjunto a un DAFO (debilidades, amenazas, fortalezas y oportunidades) con una final- idad, la "obtención de una opinión grupal fidedigna a partir de un conjunto de expertos" (Landeta Rodríguez, 2002, p. 39) y en el caso de este análisis, señalar las oportunidades.

\section{Resultados}

Una vez finalizada la etapa de envío y recogida de datos mediante los cuestionarios de ambas rondas realizados entre el 01/05/2015 y el 30/07/2015, ya que de 55 cuestionarios recibidos, pasamos a recibir en la segunda solo 12, los cuales contenían prácticamente la posición inicial e incluso alguno no respondió ya que se le explicó en el procedimiento que en caso de no hacerlo en las rondas sucesivas, se mantendrían las valoraciones iniciales. A partir de ahí se pasó a analizar cualitativamente los datos recibidos de las respuestas abiertas:

1) Cualitativos, en este caso se ha elaborado un análisis de las oportunidades con la finalidad de conocer el sector textil y su cadena de producción/consecuencias globales mediante 55 que han participado. Se han analizado de manera incisiva todas y cada una de las posiciones de nuestros expertos participantes ya que a partir de su síntesis, atrevimiento y transversalidad recogida en sus respuestas hemos podido conocer las oportunidades del sistema textil, en muchos casos siendo futuras. Se ha entendido la lógica de los tiempos asociados a la velocidad de la moda textil y a los avances que en muchos casos se traducen en mejoras y servicios en tiempo real, sino tu competidor lo hará antes y mejor que tú. A modo de ejemplo, en Bangladesh existen más de 4.500 empresas textiles dispuestas a satisfacer prácticamente cualquier requerimiento por parte de las ET textiles. 


\section{Resultados cualitativos DAFO (oportunidades) en relación al sector textil}

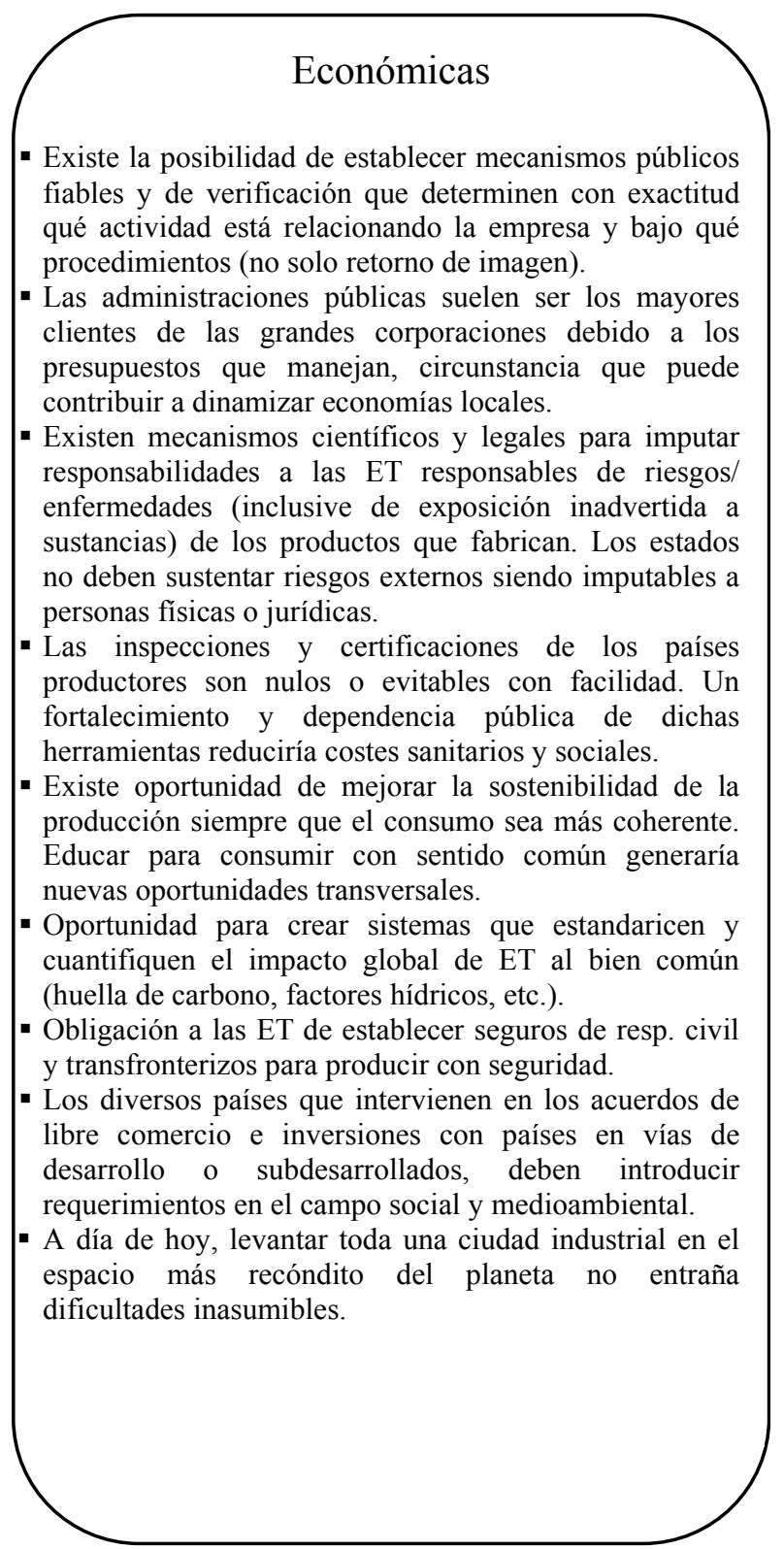

\section{Políticas, legales y normativas}

- La OIT tiene mecanismos para gestionar la adhesión/ratificación de los estados nacionales para que a la vez desarrollen mecanismos de control, aplicación de penas y sanciones no solo para los estados sino también para las empresas.

- Desarrollo de mecanismos de carácter obligatorio que den una adecuada cobertura a los derechos laborales y medioambientales básicos de todos los trabajadores, con independencia del país en el que desarrollen sus actividades, condición necesaria para que la RSE despliegue sus funciones.

- Existencia de diferentes canales/medios como inversores, trabajadores, sindicatos, ONG's, etc., para conocer si las empresas están cumpliendo con la legalidad del compromiso adquirido.

- Existen alrededor de 200 convenios de la OIT (parte del trabajo hecho), falta compromiso de gobiernos, ET y entidades supranacionales.

- Los estándares de RSC son más altos cuanto más desarrollado, civilizado y por tanto, ético y responsable es un país. Circunstancia aprovechable a la hora de articular determinadas mejoras a otros países menos desarrollados.

- Existe una oportunidad para que los trabajadores se organicen de una manera global y así hacer contrapeso al poder de las ET.

- El fortalecimiento de las instituciones en los países en donde se produce la ropa (legislación, entidades de control y rama judicial) genera oportunidades.

- Extensión de coberturas básicas de Seg. Social a países pobres aumentará la demanda de servicios.

- Las fronteras han roto el espacio de la soberanía normativa. Pensemos en modelos de trabajo internacionales.

- La construcción y desarrollo de una civilización global necesita avanzar desde regulaciones privadas de las ET a una esfera internacional. 


\section{Sociales}

- El consumidor cada vez tiene más información (y herramientas), se ha vuelto más participativo permitiéndole evaluar y actuar en tiempo real.

- El consumo responsable de productos mejorará los procedimientos industriales - como trazabilidad - con el compromiso serio de instancias internacionales y gobiernos.

- Si las multinacionales textiles se comportaran en terceros países de la misma manera que lo hacen en los suyos propios incluyendo toda la cadena de valor en relación a estándares y regulaciones, se podrían evitar muchos desastres y ahorrar costes.

- Existe la posibilidad de cambiar hacia una empresa más social aunque condicionado este cambio a la evolución del ser humano.

- El compromiso de las marcas para mejorar el los derechos de los trabajadores sobre la cadena de producción crea valor compartido.

- Existe la posibilidad de establecer un Market Place, lugar donde se concentren proveedores que cumplan las reglas laborales a nivel global.

- Además del impacto social o retorno de imagen, se puede empezar a valorar el bienestar social y la mejora ambiental.

- La RSC para superar las limitaciones (unilateralidad, marketing empresarial) debe de incluir mecanismos vinculantes que la doten de eficacia y credibilidad, los Acuerdos Marco Globales son una vía esencial. El reforzamiento de esta vía podría permitir su exigibilidad real ante los tribunales. Existe la posibilidad de elaborar una RSE vinculante a través de los acuerdos de la OMC.

- Las empresas con sistemas y medidas que humanizan el trabajo y las condiciones laborales son las que tienen más futuro.

- Existe la posibilidad de incluir criterios éticos en pliegos de condiciones públicos.

- Se espera que las sociedades en desarrollo articulen sus propios mecanismos de defensa de sus derechos.

- Las compras públicas pueden ser un buen instrumento para introducir condicionantes sociales o medioambientales, con un efecto directo en el proceder de administraciones y empresas que va más allá del puro efecto de imagen

\section{Tecnológicas y ambientales}

- El avance lógico de la tecnología puede traer consigo desarrollos textiles más limpios y éticos.

- El aumento de tecnología y normas genera nuevos productos (a medida, de seguridad, ropa inteligente) además de nuevos clientes.

- Hoy en día existe tecnología y productos químicos no agresivos con el medio ambiente que consiguen similares efectos que los tradicionales (ej. tintes naturales o envejecimiento de pantalones 'jeans').

- El principio de precaución aumenta la innovación y el desarrollo industrial.

- Es posible conocer todos los eslabones de la cadena de valor para determinar en qué condiciones se han producido esos bienes (trazabilidad). Esos mecanismos pueden ser articulados por las multinacionales sin suponerles un excesivo costo $y$ generar certidumbre.

- Los modelos laborales del futuro serán modelos de colaboración, en donde es el cliente final quien decide quien fabrica su ropa a través de internet, que componentes son utilizados en su realización, quien la transporta, etc.

- Oportunidad para reciclar y reutilizar lo que ya está producido para transformarlo en materia prima y cerrar el ciclo.

- El TEDH (Estrasburgo) o el Tribunal Internacional de La Haya podrían resolver el incumplimiento de derechos fundamentales incluyendo el medio ambiente sin necesidad de crear otro organismo (más allá de las herramientas de la OIT).

- Dentro del nuevo modelo de mercado de trabajo, destacarán el autoempleo y las microempresas con posibilidades de acceder a nuevos mercados con bajas barreras de entrada gracias a la tecnología.

- Explicar a los órganos reguladores (de productos tóxicos) las correlaciones emergentes existentes para re-evaluar el listado de sustancias. Pruebas in vitro o en células pueden determinar rápidamente actividades sospechosas para la salud.

- Las universidades tienen la oportunidad de involucrar dentro de su formación los valores humanos. La brecha entre los trabajadores de cuello blanco y cuello azul no debería existir, ambos son esenciales para que el modelo funcione. 


\section{Conclusiones}

Las empresas, además de legitimidad jurídica para desarrollar una actividad, deben ganarse la legitimidad para hacerlo mediante la correcta ponderación de fuerzas entre la normativa obligatoria y normativa o recomendación voluntaria pujante (entre la que se encuentran los deseos de los consumidores y entorno) siendo estas además una oportunidad de negocio pujante. No pueden pasarse por alto las evidencias señaladas por la (OIT, 2015, p. 107) donde en relación a la RSE del sector global de la confección señala que "Estas iniciativas unilaterales, voluntarias y no vinculantes han fracasado estrepitosamente a la hora de mejorar salarios o jornadas de trabajo o asegurar el respeto del derecho de los trabajadores de organizarse en un sindicato", lo que pone de manifiesto que los agentes necesarios para producir y sus relaciones centrípetas son complementos necesarios (empresa-sindicatos-gobiernos-organizaciones internacionales-ONG's) y no sustitutivos.

Según diferentes estudios como los de (Gildia, 1995) y (Zaman, Yamin, \& Wong, 1996) los consumidores prefieren la utilización de productos de compañías que invierten y promueven acciones de protección medioambiental además de tener un buen comportamiento con la sociedad. Para (Carroué, 2012, p. 84) "Las empresas transnacionales se ven forzadas a revisar su estrategia debido a la importancia creciente de los países emergentes, a la crisis y a la aparición de una opinión pública mundial molesta por sus prácticas". Es en ese contexto donde las ET tienen todos los elementos posibilitadores a su favor para generar valor en equidad a la vez de abrir un arcoíris de oportunidades que debe de comprenderse desde su raíz destacando:

1) Producción sostenible, para (OrganicCottonColours, 2015) "El consumidor cada vez está más informado y esto conlleva que exige más información. Cuando esto sucede el consumo masivo de artículos que no aportan nada al planeta en lo social o medioambiental irá disminuyendo" todo ello sumado a las acciones de castigo que no harán otra cosa que obligar a las empresas a mejorar sus estándares. Lo que es legal puede no ser suficiente para buena parte de la sociedad. Para el filósofo (Sádaba, 2015) "Un consumo consciente y sostenible puede transformar la orientación sociopolítica del mundo", ahora bien, "todo esto exigirá movimientos sociales serios y una constante pedagogía", paradigma que está lejos de concretarse ya que como señala (Bonet, 2015) "El cambio social ante las nuevas perspectivas, creo, precisa también de un cambio en las estructuras de producción y de prestación de servicios (socialización o economía colaborativa) que no creo que los grandes conglomerados industriales y sus lobistas estén dispuestos a permitir". Para (Porter \& Kramer, 2006, p. 7) "Si los gobiernos, las ONG y otros participantes en la sociedad civil debilitan la capacidad de las empresas para operar productivamente, pueden ganar batallas pero perderán la guerra, al degradarse la competitividad corporativa y regional, estancarse los salarios, desaparecer los trabajos y evaporarse la riqueza que paga impuestos y respalda las contribuciones sin fines de lucro.", es necesario comprender que el camino de la competitividad sostenible (y coherente) hará disfrutar de mayores beneficios a la organización (y a la sociedad), por tanto la desviación en su conducta para conseguir sus legítimos fines generará un quebrantamiento de la confianza entre empresa y consumidores además una falta de verosimilitud en las políticas de RSC tanto por acción como por omisión.

2) Competitividad, entendida como "la capacidad para, rivalizando con otras empresas, conseguir alcanzar una posición competitiva favorable, que permita obtener un desempeño superior al de los competidores" (Aragón \& Rubio., 2005, p. 37). En el contexto textil, nada tienen que ver los sofisticados cuarteles generales y metodologías de las empresas occidentales con muchos de los talleres asentados en países en vías de desarrollo. Bien es cierto que la producción ha aumentado progresivamente en los últimos 20 años y vestir bien ya no es solo objeto de los bolsillos más pudientes ¿pero es eso suficiente? Lamentablemente no, la sociedad reclama igualdad en todas sus vertientes, y en el ámbito empresarial esta debe de ser compatible con la productividad y la competitividad ya que si no corremos el riesgo de volver a la casilla de salida pero esta vez en otro país más pobre aun, por tanto de abismo en abismo. Recordemos que China ha dejado de "ser rentable" para muchas empresas debido al aumento de su salario mínimo y al establecimiento de sindicatos, para (Rios, 2012) China "Cada vez será menos el paraíso de la mano de obra barata. Basta constatar los aumentos salariales de los últimos meses en algunas zonas costeras y el proceso de deslocalización de empresas en favor de otros países del sudeste asiático. Ahora mismo, el objetivo de las autoridades es un nuevo modelo de desarrollo que eliminará progresivamente la elevada significación que antaño tenía el llamado dumping social. Los aumentos salariales son del orden del treinta por ciento o más en las zonas costeras del país, donde se requiere una mano de obra cualificada". Las sociedades con menor renta, en este caso lugares donde se asientan los talleres textiles, están deseosos de poder comprar los bienes y servicios que en muchos casos producen. Solo existe un camino para la mejora continua más allá del laboratorio e ingenierías fiscales, la coexistencia pacífica 
entre empresas y territorios lejanos con la finalidad de dinamizar las economías locales y así desplegar una cobertura real que ayude a cubrir las necesidades de la sociedad a nivel global.

3) Procesos de innovación, para (Cardona, 2012, pp. 32-33) "mediante innovación organizacional algunos grupos empresariales han desarrollado mecanismos para generar impacto social positivo, y lo han hecho creando su propio modelo, con un componente filantrópico." A la vez que "existen unos nexos fuertes entre innovación y responsabilidad social, que esos nexos continúan en construcción, y cada vez es más complejo el papel integrador entre los dos campos, porque ello exige pluralismo, apertura, alianzas entre grupos humanos muchas veces antagónicos, tanto desde el punto de vista económico como desde el punto de vista político." Por ello, es necesario desarrollar e implantar adecuadamente los procesos de innovación como han desarrollado muchas empresas para la generación de bienestar en equidad, evitando exclusión o disparidades entre empresas y sociedad. Pere Prat, ex Director General de Vivesa ${ }^{14}$, destaca que "pocas actividades retribuyen mejor a los innovadores, sean de producto o de procesos que el textil-vestuario. Así lo atestiguan éxitos como los de Armani, Valentino, Calvin Klein o Ralp Lauren, o de procesos como Amancio Ortega o Isaac Andic. Un hecho similar se repite entre empresas que han sabido crear nuevas materias primas como la Lycra, Nylon, Poliéster o Gore Text, cada uno de los cuales han dado pie a periodos de innovación".

4) Asumir responsabilidad —no solo empresarial— por parte de los gobiernos a través de sistemas de compras públicas evaluando a las cadenas de producción y sus contratistas (Schulten, Alsos, Burgess, \& Pedersen, 2012) es algo que pese a ser necesario todavía no está generalizado. Para (Martínez, 2008) "la adopción y la promoción de la responsabilidad social corporativa por parte del gobierno chino puede mirarse con recelo. En primer lugar, debemos preguntarnos hasta qué punto el consumidor chino está realmente al tanto del significado y la importancia de la responsabilidad social corporativa (algo considerado como fundamental para el desarrollo de la idea en los países occidentales)."
Aunque en muchos momentos pueda parecer que una empresa de manera aislada puede implantarse en un territorio abstrayéndose del entorno que le rodea o limitándose a importar lo que necesita desde su empresa matriz hasta cualquier base de operaciones (taller textil), puede que ese no sea el mejor camino a la hora de desarrollar políticas de RSC. El conocimiento y entendimiento de todos los agentes implicados en un proceso empresarial así como de las instituciones que la rodean (ayuntamientos, provincias, cantones, federaciones, países, gobiernos, religión, costumbres, etc.) a través de mecanismos de obligado cumplimiento son fundamentales para desarrollar la actividad industrial con éxito ya que la comunicación debe de ser fluida entre las diferentes administraciones para generar un desarrollo efectivo y equilibrado de políticas comerciales y legales.

5) Los costes de transporte internacional para las ET son muy asequibles debido al volumen de productos que se gestionan a través de grandes cargueros mercantes $^{15}$. Pero este descenso en el precio del transporte -acompañado de gran oferta existente junto al descenso del precio del petróleo-y las telecomunicaciones no es simétrico para todos los ciudadanos y países. Recientemente se ha difundido través de la Universidad Saint-Louis de Bruselas un estudio ${ }^{16}$ que analiza 25 operadores postales a nivel europeo, poniendo de manifiesto la disparidad de precios para los ciudadanos dependiendo del país y paquete que se envíe teniendo diferente coste si se envía el mismo bulto de Bélgica a Portugal o de Portugal a Bélgica. Según el comisario de la UE para el Mercado Único Digital, Andrus Ansip "Los elevados precios y la ineficiencia de la entrega transfronteriza de paquetes disuade de vender o comprar en otros países de la UE. Esto significa que el comercio electrónico no se está usando en su máximo potencial ${ }^{17}$ ", esta circunstancia debe de ser mejorada pero no a base de repercutir los costes del transporte - de las compras - a talleres que podrían vender el alma al diablo por no perder un pedido. La falta regulación y control de esas situaciones crea auténticos dramas en familias que trabajando de sol a sol -y los nublados- no son capaces de subsistir en determinados territorios de países desfavorecidos. Es evidente la necesaria regulación

\footnotetext{
${ }^{14}$ Tejiendo el futuro. Conferencia impartida en el Gremio Textil de Sabadell, noviembre del 2008. Información extraida del documento de trabajo Previsiones sobre la evolución del sector textil/confección en el horizonte de 2015, p.52 elaborado por el Observatorio Industrial del sector Textil/Confección.

${ }^{15}$ El 'McKinney Möller', de 398 metros de eslora y 58 de manga, puede llevar 18.000 contenedores: El barco más grande del Mundo

16 "Econometric study on parcel list prices", estudio elaborado por los profesores A. Claes y W. Vergote a través de la Universidad Saint-Louis de Bruselas para la Comisión Europea y publicado el 22/12/2015.

17 “El elevado precio de los envíos al extranjero lastra el comercio 'on line'”, información elaborada por B. Domínguez a través de Elpaís.com del 22/12/2015
} 
del transporte postal (paquetería) - ya que la autorregulación de las compañías postales no funcionacircunstancia que de ser normalizada evitando la lucha fratricida entre talleres textiles a la vez que generaría certidumbre al igual que pasa a modo de ejemplo con el tabaco, medicamentos, etc., que tienen mismos precios en todos los establecimientos. La mejora de esta situación sería un salto cualitativo para las empresas y consumidores, pudiéndose dedicar las primeras a mejorar otras carencias en lugar de estar pendientes continuamente de trabajar a través de monopsonios generando dumping social.

6) Involucración de la sociedad, cuanto mayor sea su implicación a la hora de escoger los productos que compran los consumidores -y administraciones públicas- prácticas más responsables se desarrollarán debido a la presión indirecta sobre su conducta. Para (Mcdonald \& Rundle-Thiele, 2008) los clientes priorizan las distintas dimensiones de la RSC en relación a sus gustos o condicionamientos sociales que estiman más convenientes, o según (Zaman, Yamin, \& Wong, 1996, pp. 20-21) los consumidores prefieren la utilización de productos de compañías que invierten en acciones de protección medioambiental además de tener un buen comportamiento con la sociedad. Existen sociedades (o segmentos de las mismas) que priorizan la compra de productos a precios irrisorios, siendo presumible que su elaboración se ha realizado en países cuyas condiciones laborales no son las más deseadas para sus trabajadores. Con el gesto-acción de compra por parte de las administraciones consumidores y usuarios, se puede ayudar a que las empresas corrijan sus conductas. La mayoría de la sociedad está dispuesta a pagar algo más por un producto elaborado en condiciones dignas, con derechos laborales y humanos apropiados, además de utilizar técnicas responsables en cualquier parte del proceso de elaboración de la prenda y lo que es más importante, obligando a las empresas a ser responsables para generar ventajas competitivas.

7) Presión social de la industria de la moda, hablar de ella sin el análisis de (Lipovetsky, 2004) sería más que atrevido ya que hoy en día el banalizado término "ir a la moda" esconde tras de sí un fenómeno multidimensional en el que se asemejan nuestros cambios de vida, tanto para bien como para mal. La sociedad está en continuo movimiento al igual que nuestras necesi- dades (más allá de que algunas hayan sido creadas artificialmente). En una reciente entrevista a (Hilfiger, 2015) señalaba que "Hay demasiadas tiendas. Hay demasiada ropa. Por eso es importante crear algo nuevo, fresco y diferente. Porque si seguimos haciendo lo mismo una y otra vez, la gente se aburrirá. La moda que no cambia muere". ¿Por qué no utilizar estas oportunidades para crear sociedades más prosperas?

8) Los usuarios tienen el deber y el derecho de conocer los productos que compran y saber en qué condiciones se han elaborado. No existe excusa para no conocer el $100 \%$ de los productos utilizados en la elaboración de las prendas así como toda la cadena de producción que interviene en la elaboración de las prendas (OIT, 2015 , p. 106). No se sostiene la excusa del secreto empresarial ya que se pueden articular funciones en organismos existentes con el consabido deber de sigilo - en cuanto a la información tratada - a la hora de poder regular la legalidad dentro de la cadena de producción y así mejorar su ética empresarial, además de ser una palanca de cambio a través de la eliminación de prácticas corruptas —evitando paralelamente desgracias- a través de las cuales se aprovechan oscuras legislaciones para producir de manera ilegal. Técnicamente es posible insertar un simple código qr en cualquiera de las etiquetas de las prendas y así conducirnos a un documento online con las especificaciones éticas y productivas necesarias de la cadena de valor.

Producir en un marco de igualdad debe de ser obligatorio. El miedo de una empresa a ser multada o manchar su reputación debido a los riesgos en la cadena de valor o por el uso de sustancias químicas que aun siendo legales, estudios independientes advierten de sus riesgos hoy en día ya no pueden ser asumibles. La implantación de un código o mecanismo a nivel global vinculante es una de las posibles soluciones de mejora siempre que no sea elaborado por ET con intereses partidistas o gobiernos ávidos de inversión extranjera — siendo esta bienvenida pero no a cualquier precio-.

9) Las Agencias de calificación (Teitelbaum, 2013) y certificación $^{18}$ (Navarro, 2013) en las que se apoyan ciertos inversores, ET, normas e incluso sentencias "no es lo mismo una catástrofe certificada a otra que no lo está", en la actualidad no son más que predictores y requisitos en muchos casos impuestos o semi necesarios para producir. La desposesión de facultades a

\footnotetext{
18 "Se ha generado una industria de la auditoría social de millones de dólares, lo que significa que, pese a la falta de resultados, hay tanto dinero invertido que muchas empresas seguirán apostando por alcanzar cambios incrementales mediante mejoras en los enfoques existentes. Sin embargo, como el modelo de auditoría y cumplimiento de las normas se centra en el desempeño individual de las fábricas, sin identificar o enfrentar las causas de fondo y las barreras sistémicas, esos esfuerzos seguirán siendo ineficaces" a través del documento de trabajo de la OIT titulado Trabajo decente en las cadenas mundiales de suministro, p.109 elaborado por varios autores entre ellos J. Holdcroft autora de la cita. V.7, №1-2, año 2015.
} 
los Estados debe de ser estudiada (reservándose sus competencias como indelegables de por vida más allá de acuerdos puntuales) y no únicamente externalizándose $^{19}$ al mejor postor. Cabe la pena analizar ¿cuál es la diferencia entre no estar calificado y estar (des)calificado?

10) Los AMI (acuerdos marco internacionales) son una apuesta decidida de muchas multinacionales textiles en favor del cumplimiento de un mínimo de condiciones para trabajadores que desarrollan actividad productiva - generalmente para contratistas y subcontratistas directos de las ET- en situaciones en muchos casos no muy propicias para la promoción y correcto desarrollo de derechos fundamentales. Las ET textiles y su modelo productivo han logrado - aunque de manera tibia - vincular a muchas de las federaciones sindicales internacionales con la finalidad de transformar muchos de sus códigos de conducta en AMI haciéndolos vinculantes (Michel, 2007). Hoy más que nunca es necesaria la comprensión de las complejas estructuras societarias existentes a nivel global así como elevar la coordinación intersectorial sindical de todos los integrantes de la cadena de valor y países donde operan las ET. La predisposición de muchas empresas textiles a firmar y desarrollar los AMl es un avance mientras se fomente de manera paralela el trabajo digno. No se pueden crear mecanismos sistémicos para regular las condiciones de los trabajadores cuando gran parte de los mismos ya no tiene esa cualidad pese a la firma de todo tipo de acuerdos.

\section{Bibliografia}

ANTOLÍN, M., \& CENDRERO, J. (2011). Las empresas transnacionales en la periferia capitalista. Economía política de la crisis.

ARAGÓN, A., \& RUBIO., A. (2005). Factores explicativos del éxito competitivo: El caso de las Pymes del estado de Veracruz. Contaduría y Administración.

ARIAS, M. (2003). Metodologías de investigación emergentes en economía de la empresa. Bordeaux: , Papers Proceedings 2003, XVII Congreso Nacional XIII congreso hispano-francés AEDEM,Université Montesquieu Bordeaux IV.

BECK, U. (1998). ¿Qué es la globalización? Falacias del globalismo, respuestas a la globalización. Paidós Estado y Sociedad.
BONET, J. (07 de 2015). Gestión y control de la cadena de valor dentro del sector textil en países en vías de desarrollo, hacia los límites de la logística y el outsourcing: Ética o Estética. Documentación propia extraida de un analisis DELPHI/entrevista al autor. (A. Luque, Entrevistador)

BOUZAS, R. (1994). Evaluación de acciones de integración económica. Con la colaboración de Friedrich-Ebert-Stiftung. Fundación Raúl Prebisch.

CARDONA, H. (2012). "Innovación y responsabilidad social: una reflexión sobre los puntos de encuentro". Revista Universidad \& Empresa, 13(21), 13-35.

CARROUÉ, L. (2012). "Empresas, el reino de las trananacionales". (M. Sfeir, Ed.) El Atlas de las Mundializaciones, 84 .

DEL RINCÓN, D., ARNAL, J., LATORRE, A., \& SANS, A. E. (1995). Técnicas de investigación en Ciencias Sociales. Madrid: Dikinson.

DUNNING, J. (2012). International Production and the Multinational Enterprise. RLE International Business; V.12 Routledge.

FERNÁNDEZ-BALLESTEROS, R. (1995). Evaluación de programas: una guía práctica en ámbitos sociales,educativos y de salud. Madrid: Síntesis.

FERNANDO, H., \& RIVERA, C. (2013). "Empresas y derechos humanos:¿ hacia una regulación jurídica efectiva, o el mantenimiento del status quo? Corporations and Human Rights: Towards an Effective Legal Regulation". Anuario Mexicano de Derecho Internacional, 13, 313-354.

FOLKES, V., \& KAMMIS, M. (1999). "Effects of information about firms'ethical and unethical actions on consumers'attitudes". Journal of consumer psycology, 8 (3), 243-259.

GILDIA, R. (1995). "Consumer survey confirms corporate social responsibility affects buying decisions". Public Relations Quarterly, No39, 20-21.

GRAU-RUIZ, M. (2013). "Responsabilidad social empresarial y fiscalidad internacional en relación con la inversión directa extranjera en países en desarrollo". Revista de Globalización, Competitividad y Gobernabilidad, 34-48.

\footnotetext{
${ }^{19}$ Los 10 mercados emergentes para externalizar son 1-Bulgaria, 2-Brasil, 3-Costa Rica, 4-República Checa, 5-Egipto, 6-Hungría,

7-Malasia, 8-México, 9-Portugal y 10-Vietnam. Información elaborada por K. Hopkins a través de Rancoteur el 10/12/2015.
} 
HARBULOT, C. (2012). ¿Tendencia a la relocalización? El Atlas de las Mundializaciónes, 156.

HERNÁNDEZ, L., RAMÍREZ , F., \& ÁlVAREZ, M. (2013). "Mercadotecnia: estrategia esencial para el comercio global". Observatorio de la Economía Latinoamericana, 186. Obtenido de http://www.eumed. net/cursecon/ecolat/mx/2013/mercadotecnia.html

HILFIGER, T. (17 de 02 de 2015). Tommy Hilfiger: 'La moda que no cambia muere'. (L. Blanco, Entrevistador, \& Elmundo.es, Editor) Obtenido de http:// www.elmundo.es/cultura/2015/02/17/54e2591bca4741e9468b4570.html

HUMAN RIGHTS WATCH. (12 de 03 de 2015). Camboya: Trabajadores de la industria de la confección están desprotegidos. Obtenido de http:// www.hrw.org/es/news/2015/03/11/camboya-trabajadores-de-la-industria-de-la-confeccion-estan-desprotegidos

IETTO-GILLIES, G. (2012). Transnational corporations and international production: concepts, theories and effects. Edward Elgar Publishing.

KALMANOVITZ, S. (2010). Nueva Historia Económica de Colombia. Bogotá: Taurus Historia y Universidad Jorge Tadeo Lozano.

LANDETA RODRÍGUEZ, J. (2002). El método Delphi. Una técnica de previsión del futuro. Barcelona: Ariel.

LARREA, C. (2013). Globalización y contabilidad: Algunas reflexiones en torno a la obra el trabajo de las naciones de robert B. Reich/Globalization and accounting: Some reflections about the work of nations by robert B. reich. Contabilidad y Negocios, 8(15), 95-101.

LINSTONE, H., \& TUROFF, M. (1975). The Delphi method: Techniques and applications. Reading, MA: Addison-Wesley. V.29.

LIPOVETSKY, G. (2004). El imperio de lo efímero: la moda y su destino en las sociedades modernas. Anagrama.

LOVINS, E., HUNTER, L., \& HAWKEN, P. (2013). Natural capitalism: The next industrial revolution. Routledge.

LUNA HUERTAS, P., INFANTE MORO, A., \& MARTÍNEZ , F. (2006). Los Delphi como fundamento metodológico predictivo para la investigación en sistemas de información y tecnologías de la infor- mación (Is/lt). Pixel-Bit. Revista de Medios y Educación $\mathrm{N}^{\circ} 26,89-112$.

MARTÍNEZ PIÑEIRO, E. (2003). "La técnica DELPHI como estrategia de consulta a los implicados en la evaluación de programas". Revista de Investigación Educativa, V.21, №2, 449-463.

MARTÍNEZ, P. (2008). 'He Xie She Hui', una sociedad armoniosa $i$ Es posible la responsabilidad social corporativa en China? Observatorio de la Economía y la Sociedad China, No13. Obtenido de http://www. eumed.net/rev/china/13/pnm.htm

MCDONALD, L., \& RUNDLE-THIELE, S. (2008). Corporate social responsibility and bank customer satisfaction. International Journal of Bank Marketing, V. 26.

MENGUAL, S. (2011). La importancia percibida por el profesorado y el alumnado sobre la inclusión de la competencia digital en educación Superior. Alicante: Departamento de Didáctica General y Didácticas específicas de la Facultad de Alicante.

MEYERS, H. (2012). The nationality of ships. Springer.

MICHEL, D. (2007). Acuerdo marco internacional: una herramienta para apoyar los derechos en el trabajo. OIT. Obtenido de http://www.ilo.org/global/aboutthe-ilo/newsroom/features/WCMS_080725/lang-es/index.htm

MIRALLES, O. (08 de 2015). "Gestión y control de la cadena de valor dentro del sector textil en países en vías de desarrollo, hacia los límites de la logística y el outsourcing: Ética o Estética". Documentación propia extraida de un analisis DELPHI/entrevista al autor. (A. Luque, Entrevistador)

MORO, B. (2011). Las empresas transnacionales como agentes centrales de la globalización neoliberal, elementos de discusión para una propuesta de políticas alternativas. Lan Harremanak. Revista de Relaciones Laborales.

NAVARRO, V. (23 de 05 de 2013). Lo que no se dice sobre Bangladesh. Obtenido de vnavarro.org: http:// www.vnavarro.org/? $p=8939$ \&lang $=C A$

OBSERVATORIO INDUSTRIAL DEL SECTOR TEXTIL/CONFECCIÓN. (2015). Previsiones sobre la evolución del sector textil/confección en el horizonte de 2015. Obtenido de http://www.industria.ccoo.es/ comunes/recursos/99927/308012-Prevision_sobre_ la_evolucion_del_sector_textil_-_confeccion_en_ el_horizonte_2015.pdf 
OIT. (2013). Informe Mundial sobre Salarios 2012/2013, los salarios y el crecimiento equitativo.

OIT. (2015). Trabajo decente en las cadenas mundiales de suministro. Boletín Internacional de Investigación Sindical. V.7, №1-2.

ORGANICCOTTONCOLOURS. (07 de 2015). Gestión y control de la cadena de valor dentro del sector textil en países en vías de desarrollo, hacia los límites de la logística y el outsourcing: Ética o Estética. Documentación propia extraida de un analisis DEL$\mathrm{PHI} /$ entrevista al autor. (A. Luque, Entrevistador)

PILL, J. (1971). "The Delphi method: substance, context, a critique and an annotated bibliography". Socio-Economic Planning Sciences. V.5, issue 1, 5771.

PORTER, E., \& KRAMER, M. (2006). "Estrategia y sociedad". Harvard Business Review. América Latina. V.84, Nº12, 42-56.

PULIDO, A., \& RAMIRO, P. (2011). "La Responsabilidad Social Corporativa de las multinacionales españolas en Colombia". Lan Harremanak. Revista de Relaciones Laborales, 236.

QUINTANILLA, I. (24 de 12 de 2015). "El modelo de sociedad nos hace ser consumidores compulsivos porque es lo que conviene". (T. Pinto, Entrevistador) Obtenido de http://www.eldiario.es/sociedad/consumo_compulsivo-Navidades-psicologia_del_consumidor_0 463703807.html

REINECKE, J., \& DONAGHEY, J. (2015). "After Rana Plaza: building coalitional power for labour rights between unions and (consumption-based) social movement organisations". Organization, doi, 10. Organization 22 .

RÍOS NÚNEZ, S., \& COQ HUELVA, D. (2010). El poder de la Gran Distribución en el sistema agroalimentario actual: El caso de los lácteos en Chile. Estudios sociales (Hermosillo, Son.), 18(36).

RIOS, X. (01 de 2012). "China: Desigualdad, corrupción y progreso". (S. Lopez Arnal, Entrevistador) El Viejo Topo, 288, 17. Obtenido de http://dialnet.unirioja.es/servlet/articulo?codigo $=3915723$

RUGMAN, A. (1982). New Theories of the Multinational Enterprise. London: Croom Helm.

RUIZ OLABUÉNAGA, J., \& ISPUZUA, M. (1989). La descodificación de la vida cotidiana. Bilbao: Universidad de Deusto.
SÁDABA, J. (17 de 01 de 2015). El cliente tiene la razón y el poder. Noticia elaborada por Miguel Ángel García Vega. (Elpaís, Ed.) Obtenido de http:// economia.elpais.com/economia/2015/01/15/actualidad/1421336430_884539.html

SCHULTEN, T., ALSOS, K., BURGESS, P., \& PEDERSEN, K. (2012). Pay and other social clause in European public procurement. Düsseldorf: Hans Böckler Stiftung.

STRAUSS, A., \& CORBIN, J. (1990). Basics of Qualitative Research: Grounded Theory, procedures and techniques. Sage Publications. Newbury Park, CA.

TANZI, V., \& DAVOODI, H. (1997). Corruption, public investment, and growth. IMF, Working Paper $\mathrm{N}^{\circ}$ 97/139.

TEITELBAUM, A. (22 de 04 de 2013). "La impunidad de los delincuentes económicos de las clases dominantes". Argenpress.info. Obtenido de http://www. argenpress.info/2013/04/la-impunidad-de-los-delincuentes.html

TOKMANN, V. (2012). Políticas de empleo en la nueva era económica. Economía, 23(46).

TOSCANO, R. (07 de 2015). "Gestión y control de la cadena de valor dentro del sector textil en países en vías de desarrollo, hacia los límites de la logística y el outsourcing: Ética o Estética". Documentación propia extraida de un analisis DELPHI/entrevista al autor. (A. Luque, Entrevistador)

VALLES, M. (2014). Entrevistas cualitativas. CIS-Centro de Investigaciones Sociológicas. V.32.

VARGAS HERNÁNDEZ, J. (2012). Cooperacion y conflicto entre empresas, comunidades y gobierno: nuevos movimientos sociales, el caso de Cerro de San Pedro, México. In International Conference Virtual City and Territory. Centre de Política de Sòl i Valoracions.

VÉLEZ PAREJA, I. (2002). Decisiones de inversión enfocado a la valoración de empresas. Bogotá: CEJA.

ZAMAN, M., YAMIN, S., \& WONG, F. (1996). Environmental consumerism and buying preference for green products. Proceedings of the Australian Marketing Educators Conference, 613-626. 